PROCEEDINGS OF THE

AMERICAN MATHEMATICAL SOCIETY

Volume 140, Number 9, September 2012, Pages 3127-3132

S 0002-9939(2012)11138-4

Article electronically published on January 18, 2012

\title{
RIESZ BASES OF EXPONENTIALS ON MULTIBAND SPECTRA
}

\author{
NIR LEV \\ (Communicated by Michael T. Lacey)
}

\begin{abstract}
Let $S$ be the union of finitely many disjoint intervals on $\mathbb{R}$. Suppose that there are two real numbers $\alpha, \beta$ such that the length of each interval belongs to $\mathbb{Z} \alpha+\mathbb{Z} \beta$. We use quasicrystals to construct a discrete set $\Lambda \subset \mathbb{R}$ such that the system of exponentials $\{\exp 2 \pi i \lambda x, \lambda \in \Lambda\}$ is a Riesz basis in the space $L^{2}(S)$.
\end{abstract}

\section{INTRODUCTION}

1.1. Let $S$ be the union of a finite number of bounded intervals on $\mathbb{R}$. We denote by $P W_{S}$ (after Paley and Wiener) the space of all functions $f \in L^{2}(\mathbb{R})$ whose Fourier transform

$$
\widehat{f}(x)=\int_{\mathbb{R}} f(t) e^{2 \pi i x t} d t
$$

vanishes almost everywhere outside of $S$. A discrete set $\Lambda \subset \mathbb{R}$ is called a complete interpolation set for $P W_{S}$ if the restriction operator $\left.f \mapsto f\right|_{\Lambda}$ is a bounded and invertible one from $P W_{S}$ onto $\ell^{2}(\Lambda)$. In the context of communication theory this means that $\Lambda$ provides a "stable and non-redundant" sampling of signals with spectrum in $S$.

It is well-known that the complete interpolation property of $\Lambda$ is equivalent to the Riesz basis property of the corresponding exponential system

in the space $L^{2}(S)$.

$$
E(\Lambda)=\{\exp 2 \pi i \lambda x, \lambda \in \Lambda\}
$$

If $S$ is a single interval, then a complete description of the Riesz bases $E(\Lambda)$ in $L^{2}(S)$ was given by B. S. Pavlov (1979). Much less is known, however, in the case when $S$ is the union of more than one interval. In fact, it is unknown in general whether an exponential Riesz basis in $L^{2}(S)$ exists at all. This existence has been established in the following special cases:

(i) $S$ is a finite union of disjoint intervals with commensurable lengths [2, 4.

(ii) $S$ is the union of two general intervals 8 .

For other results in the subject we refer to the survey paper 4 .

In this paper we extend the two results above and prove:

Theorem 1. Let $S$ be the union of finitely many disjoint intervals on $\mathbb{R}$. Suppose that there are two real numbers $\alpha, \beta$ such that the length of each interval belongs to $\mathbb{Z} \alpha+\mathbb{Z} \beta$. Then there is $\Lambda \subset \mathbb{R}$ such that $E(\Lambda)$ is a Riesz basis in $L^{2}(S)$.

Received by the editors February 6, 2011 and, in revised form, March 21, 2011.

2010 Mathematics Subject Classification. Primary 42C15, 94A12.

Key words and phrases. Riesz bases, multiband signals, quasicrystals.

(C)2012 American Mathematical Society 
Here $\mathbb{Z} \alpha+\mathbb{Z} \beta$ denotes the set of real numbers of the form $n \alpha+m \beta(n, m \in \mathbb{Z})$. The above-mentioned results are thus obtained as special cases of Theorem 1 .

1.2. In fact, we will prove the following more general result.

Theorem 2. Suppose that the indicator function of a set $S \subset \mathbb{R}$ can be expressed as a linear combination of indicator functions of intervals $I_{1}, \ldots, I_{N}$ whose lengths belong to $\mathbb{Z} \alpha+\mathbb{Z} \beta$, that is,

$$
\mathbf{1}_{S}(x)=\sum_{j=1}^{N} c_{j} \mathbf{1}_{I_{j}}(x), \quad\left|I_{j}\right| \in \mathbb{Z} \alpha+\mathbb{Z} \beta \quad(1 \leqslant j \leqslant N),
$$

where $\alpha, \beta \in \mathbb{R}$. Then there is $\Lambda \subset \mathbb{R}$ such that $E(\Lambda)$ is a Riesz basis in $L^{2}(S)$.

Sets with the structure (11) form a wider class than unions of disjoint intervals with lengths in $\mathbb{Z} \alpha+\mathbb{Z} \beta$. For example, one may take an interval with "holes" obtained by the removal of disjoint sub-intervals, where the interval and the removed sub-intervals have their lengths in $\mathbb{Z} \alpha+\mathbb{Z} \beta$.

A result of similar type in the periodic setting was obtained in 3 .

\section{Quasicrystals. Duality}

Our approach is inspired by the papers [6, 7] due to Matei and Meyer, who introduced the usage of so-called 'simple quasicrystals' in order to construct "universal" sets of sampling or interpolation for $P W_{S}$ spaces. Here we will use simple quasicrystals to construct complete interpolation sets for spectra $S$ with the structure (11).

Following [6, 7] we let $\Gamma$ be a lattice in $\mathbb{R}^{2}$. Consider the projections $p_{1}(x, y)=x$ and $p_{2}(x, y)=y$, and assume that the restrictions of $p_{1}$ and $p_{2}$ to $\Gamma$ are injective. Let $\Gamma^{*}$ be the dual lattice, consisting of all vectors $\gamma^{*} \in \mathbb{R}^{2}$ such that $\left\langle\gamma, \gamma^{*}\right\rangle \in \mathbb{Z}$, $\gamma \in \Gamma$.

Let $S$ be the union of disjoint semi-closed intervals,

$$
S=\bigcup_{j=1}^{\nu}\left[a_{j}, b_{j}\right), \quad a_{1}<b_{1}<\cdots<a_{\nu}<b_{\nu},
$$

and let

$$
I=[a, b)
$$

be a single semi-closed interval. Define

$$
\begin{aligned}
\Lambda(\Gamma, I) & =\left\{p_{1}(\gamma): \gamma \in \Gamma, p_{2}(\gamma) \in I\right\}, \\
\Lambda^{*}(\Gamma, S) & =\left\{p_{2}\left(\gamma^{*}\right): \gamma^{*} \in \Gamma^{*}, p_{1}\left(\gamma^{*}\right) \in S\right\} .
\end{aligned}
$$

In some sense, the quasicrystals $\Lambda(\Gamma, I)$ and $\Lambda^{*}(\Gamma, S)$ are dual to each other. This duality was observed and used by Matei and Meyer in connection with sampling and interpolation. In the present context of exponential Riesz bases the duality can be formulated as follows. 
Lemma 1. The following two properties are equivalent:

(i) $E(\Lambda(\Gamma, I))$ is a Riesz basis in $L^{2}(S)$;

(ii) $E\left(\Lambda^{*}(\Gamma, S)\right)$ is a Riesz basis in $L^{2}(I)$.

The proof of Lemma 1 is along similar lines as in the paper [7] (Sections 67 ), but in our case there is an additional point concerned with the requirement that the intervals in (2) and (3) should be semi-closed. One may see that this point is indeed significant by keeping in mind that the Riesz basis property of the exponential systems $E(\Lambda(\Gamma, I))$ and $E\left(\Lambda^{*}(\Gamma, S)\right)$ is not preserved upon either the addition or removal of any element.

For a proof of the duality lemma in the periodic setting, see [3, Section 2].

\section{Proof of Theorem 2}

There is no loss of generality in assuming that the numbers $\alpha, \beta$ are linearly independent over the rationals. Moreover, by rescaling we may restrict ourselves to the case when

$\alpha$ is an irrational number and $\beta=1$.

3.1. Define a lattice

$$
\Gamma=\{(n(1+\alpha)-m, m-n \alpha): n, m \in \mathbb{Z}\},
$$

and let $I=[0$, mes $S)$ be an interval whose length coincides with the Lebesgue measure of $S$. We will prove that the exponential system $E(\Lambda(\Gamma, I))$ is a Riesz basis in $L^{2}(S)$. According to Lemma 1 it will be sufficient to show that the system $E\left(\Lambda^{*}(\Gamma, S)\right)$ is a Riesz basis in $L^{2}(I)$.

It is easy to check that the set $\Lambda^{*}(\Gamma, S)$ may be partitioned as follows:

$$
\Lambda^{*}(\Gamma, S)=\bigcup_{n \in \mathbb{Z}} \Lambda_{n}, \quad \Lambda_{n}=(S \cap(n \alpha+\mathbb{Z}))+n
$$

(where some of the sets $\Lambda_{n}$ may be empty). Let $\left\{s_{n}\right\}$ be a sequence of integers such that $s_{n}-s_{n-1}=\# \Lambda_{n}$, and choose an enumeration $\left\{\lambda_{j}, j \in \mathbb{Z}\right\}$ of the set $\Lambda^{*}(\Gamma, S)$ such that

$$
\Lambda_{n}=\left\{\lambda_{j}: s_{n-1} \leqslant j<s_{n}\right\} \quad(n \in \mathbb{Z}) .
$$

In order to prove that $E\left(\Lambda^{*}(\Gamma, S)\right)$ is a Riesz basis in $L^{2}(I)$, it will be sufficient, by a theorem of Avdonin [1], to check that the following three conditions hold:

(a) $\left\{\lambda_{j}\right\}$ is a separated sequence, $\inf _{j \neq k}\left|\lambda_{j}-\lambda_{k}\right|>0$.

(b) $\sup _{j}\left|\delta_{j}\right|<\infty$, where $\delta_{j}=\lambda_{j}-j / \operatorname{mes} S$.

(c) There is a constant $c$ and a positive integer $N$ such that

$$
\sup _{a \in \mathbb{Z}}\left|\frac{1}{N} \sum_{j=a+1}^{a+N} \delta_{j}-c\right|<\frac{1}{4 \operatorname{mes} S} .
$$

Condition (a) can easily be verified directly from the definition of $\Lambda^{*}(\Gamma, S)$. 
3.2. We will next show that (b) holds. Consider the bounded, piecewise constant function

$$
\phi(x)=\sum_{k \in \mathbb{Z}} \mathbf{1}_{S}(x-k) .
$$

This function is 1-periodic and hence may be viewed as a function on the circle group $\mathbb{T}=\mathbb{R} / \mathbb{Z}$. The assumptions (11), (2) and (4) now provide the following representation for $\phi$.

Lemma 2. There is a bounded, piecewise linear function $\psi: \mathbb{T} \rightarrow \mathbb{R}$ such that

$$
\phi(x)=\operatorname{mes} S+\psi(x)-\psi(x-\alpha), \quad x \in \mathbb{T} .
$$

For a proof of Lemma 2] see [3, Lemma 3.2]. Now observe that by (5) we have

$$
s_{n}-s_{n-1}=\# \Lambda_{n}=\phi(n \alpha) .
$$

It follows from (77) and Lemma 2 that

$$
s_{n}=n \operatorname{mes} S+\psi(n \alpha)+\text { const . }
$$

Given $j$ there is $n=n(j)$ such that $\lambda_{j} \in \Lambda_{n}$ or, equivalently, such that $s_{n-1} \leqslant j<$ $s_{n}$. Then

$$
\delta_{j}=\lambda_{j}-\frac{j}{\operatorname{mes} S}=\left(\lambda_{j}-n\right)+\left(n-\frac{s_{n}}{\operatorname{mes} S}\right)+\frac{s_{n}-j}{\operatorname{mes} S} .
$$

It thus follows from (5), (7) and (8) that $\sup _{j}\left|\delta_{j}\right|<\infty$, which confirms condition (b) above.

3.3. It remains to establish (c), In order to show that (6) holds, we will first obtain a simple expression for the sum $\sum \delta_{j}$ where $j$ goes through the interval $s_{n-1} \leqslant j<s_{n}$. Indeed,

$$
\sum_{j=s_{n-1}}^{s_{n}-1} \delta_{j}=\sum_{j=s_{n-1}}^{s_{n}-1}\left(\lambda_{j}-n\right)-\sum_{j=s_{n-1}}^{s_{n}-1}\left(\frac{j}{\operatorname{mes} S}-n\right) \stackrel{\text { def }}{=} S_{1}(n)-S_{2}(n) .
$$

We evaluate each one of the sums $S_{1}(n), S_{2}(n)$ separately. First we observe that by (5),

$$
S_{1}(n)=\sum_{k \in \mathbb{Z}}(n \alpha-k) \mathbf{1}_{S}(n \alpha-k) \stackrel{\text { def }}{=} \tau_{1}(n \alpha) .
$$

Second, by a direct calculation and using (7) and (8), we find that

$$
\begin{aligned}
S_{2}(n) & =\left(s_{n}-s_{n-1}\right)\left(\frac{s_{n-1}+s_{n}-1}{2 \operatorname{mes} S}-n\right) \\
& =\frac{\phi(n \alpha)\left(\psi(n \alpha)-\frac{1}{2} \phi(n \alpha)+\text { const }\right)}{\operatorname{mes} S} \stackrel{\text { def }}{=} \tau_{2}(n \alpha) .
\end{aligned}
$$

We conclude that for an appropriately defined function $\tau: \mathbb{T} \rightarrow \mathbb{R}$ (bounded and piecewise continuous) we have

$$
\sum_{j=s_{n-1}}^{s_{n}-1} \delta_{j}=\tau(n \alpha), \quad n \in \mathbb{Z}
$$


3.4. Now we can finish the proof of (c) above. Given $a \in \mathbb{Z}$ and a positive (large) integer $N$ there are $n=n(a)$ and $r=r(a, N)$ such that

$$
s_{n-1} \leqslant a<s_{n}, \quad s_{n+r-1} \leqslant a+N<s_{n+r} .
$$

Since the sequence $\left\{\delta_{j}\right\}$ is bounded and due to (9) we have

$$
\sum_{j=a+1}^{a+N} \delta_{j}=\sum_{j=s_{n-1}}^{s_{n+r-1}-1} \delta_{j}+O(1)=\sum_{k=n}^{n+r-1} \tau(k \alpha)+O(1) .
$$

The points $\{n \alpha\}$ are well-distributed on the circle $\mathbb{T}$ (since $\alpha$ is irrational), and hence

$$
\sum_{k=n}^{n+r-1} \tau(k \alpha)=r \int_{\mathbb{T}} \tau(x) d x+o(r), \quad r \rightarrow \infty,
$$

uniformly with respect to $n$. Since (7) and (8) imply that $N=r$ mes $S+O(1)$, we get

$$
\frac{1}{N} \sum_{j=a+1}^{a+N} \delta_{j}=\frac{1}{\operatorname{mes} S} \int_{\mathbb{T}} \tau(x) d x+o(1), \quad N \rightarrow \infty,
$$

with the $o(1)$ uniform with respect to $a$. This implies [c) and so Theorem 2 is proved.

\section{REMARKS}

We have constructed Riesz bases of exponentials with real frequencies for multiband spectra subject to the diophantine condition (1). We would like, though, to comment on one result which is not covered by our theorems above. In the paper [8] existence of such Riesz bases was proved under certain non-discrete conditions on the lengths of the gaps between the intervals. The restrictions obtained there are rather severe; however, the result indicates that diophantine restrictions are not necessarily natural ones in the problem.

We also refer the reader to the paper [5] where the authors construct, for any finite union of intervals, a Riesz basis of exponentials with complex frequencies lying in a horizontal strip along the real axis.

\section{ACKNOWLEDGEMENT}

The author thanks Kristian Seip for reading an earlier version of this paper.

\section{REFERENCES}

[1] S. A. Avdonin, "On the question of Riesz bases of exponential functions in $L^{2}$ " (in Russian), Vestnik Leningrad Univ. 13 (1974), 5-12. English translation in Vestnik Leningrad Univ. Math. 7 (1979), 203-211. MR0361746 (50:14191)

[2] L. Bezuglaya, V. Katsnelson, "The sampling theorem for functions with limited multi-band spectrum", Z. Anal. Anwendungen 12 (1993), 511-534. MR.1245936 (94k:94003)

[3] G. Kozma, N. Lev, "Exponential Riesz bases, discrepancy of irrational rotations and BMO", J. Fourier Anal. Appl. 17 (2011), 879-898.

[4] Yu. Lyubarskii, K. Seip, "Sampling and interpolating sequences for multiband-limited functions and exponential bases on disconnected sets", J. Fourier Anal. Appl. 3 (1997), 597-615. MR.1491937 (99f:30007)

[5] Yu. Lyubarskii, I. Spitkovsky, "Sampling and interpolation for a lacunary spectrum", Proc. Roy. Soc. Edinburgh Sect. A 126 (1996), 77-87. MR.1378833(97b:41004) 
[6] B. Matei, Y. Meyer, "Quasicrystals are sets of stable sampling", C. R. Acad. Sci. Paris, Ser. I 346 (2008), 1235-1238. MR2473299 (2010g:94050)

[7] B. Matei, Y. Meyer, "Simple quasicrystals are sets of stable sampling", Complex Var. Elliptic Equ. 55 (2010), 947-964. MR2674875

[8] K. Seip, "A simple construction of exponential bases in $L^{2}$ of the union of several intervals", Proc. Edinburgh Math. Soc. 38 (1995), 171-177. MR.1317335 (96c:42006)

Department of Mathematics, Weizmann Institute of Science, Rehovot 76100, Israel

E-mail address: nir.lev@weizmann.ac.il 